\title{
Probing quantum phases of ultracold atoms in optical lattices by transmission spectra in cavity quantum electrodynamics
}

\author{
IGOR B. MEKHOV ${ }^{1,2 * \dagger}$, CHRISTOPH MASCHLER ${ }^{1 *}$ AND HELMUT RITSCH ${ }^{1 * \dagger}$ \\ ${ }^{1}$ Institute for Theoretical Physics, University of Innsbruck, Technikerstr. 25, 6020 Innsbruck, Austria \\ ${ }^{2}$ St Petersburg State University, V. A. Fock Institute of Physics, Ulianovskaya 1, 198504 St Petersburg, Russia \\ *These authors contributed equally to this work \\ †e-mail: Igor.Mekhov@uibk.ac.at; Helmut.Ritsch@uibk.ac.at
}

Studies of ultracold gases in optical lattices provide a means for testing fundamental and application-oriented quantum manybody concepts of condensed-matter physics in well controllable atomic systems ${ }^{1}$; examples include strongly correlated phases and quantum-information processing. Standard methods to observe quantum properties of Bose-Einstein condensates are based on matter-wave interference between atoms released from traps $^{2-6}$, a method that ultimately destroys the system. Here, we propose a new approach on the basis of optical measurements that conserves the number of atoms. We prove that atomic quantum statistics can be mapped on transmission spectra of high- $Q$ cavities, where atoms create a quantum refractive index. This can be useful for studying phase transitions ${ }^{7}$ - for example, between Mott insulator and superfluid states - as various phases show qualitatively distinct light scattering. Joining the paradigms of cavity quantum electrodynamics and ultracold gases could enable conceptually new investigations of both light and matter at ultimate quantum levels. We predict effects accessible in experiments that recently became possible ${ }^{8}$.

All-optical methods to characterize atomic quantum statistics were proposed for homogeneous Bose-Einstein condensates ${ }^{9-13}$ and some modified spectral properties induced by Bose-Einstein condensates were attributed to collective emission ${ }^{9,10}$, recoil shifts ${ }^{12}$ or local field effects ${ }^{14}$.

We show a completely different phenomenon directly reflecting atom quantum statistics due to state-dependent dispersion. More precisely, the dispersion shift of a cavity mode depends on the atom number. If the atom number in some lattice region fluctuates from realization to realization, the modes get a fluctuating frequency shift. Thus, in the cavity transmission spectrum, resonances appear at different frequencies directly reflecting the atom number distribution function. Such a measurement allows us to calculate atomic statistical quantities, for example, mean value and variance reflected by spectral characteristics such as the central frequency and width.

Different phases of a degenerate gas possess similar meanfield densities but different quantum amplitudes. This leads to a superposition of different transmission spectra, which, for example, for a superfluid (SF) state consist of numerous peaks reflecting the discreteness of the matter field. Analogous discrete spectra reversing the role of atoms and light, thus reflecting the photon structure of electromagnetic fields, were obtained in

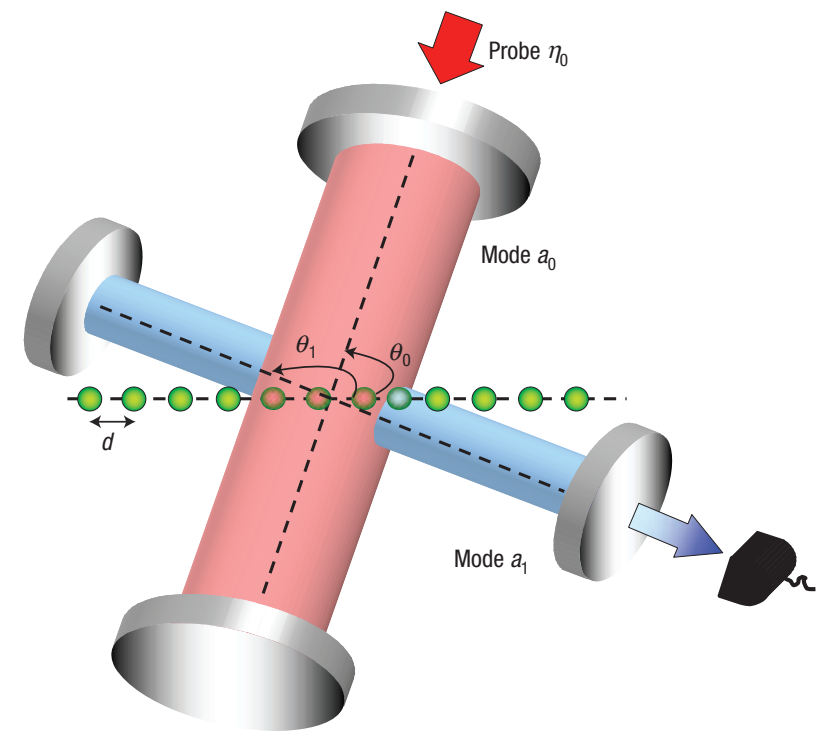

Figure 1 Schematic diagram of the set-up. Atoms are periodically trapped in an optical lattice created by laser beams, which are not shown. In addition, the atoms are illuminated by two light modes at angles $\theta_{0}$ and $\theta_{1}$ with respect to the lattice axis.

cavity quantum electrodynamics (QED) with Rydberg atoms ${ }^{15}$ and solid-state superconducting circuits ${ }^{16}$. A quantum phase transition towards a Mott insulator (MI) state is characterized by a reduction of the number of peaks towards a single resonance, because atom number fluctuations are significantly suppressed ${ }^{17,18}$. As our detection scheme is based on non-resonant dispersive interaction independent of a particular level structure, it can also be applied to molecules ${ }^{19,20}$.

We consider the quantized motion of $N$ two-level atoms in a deep periodic optical lattice with $M$ sites formed by far offresonance standing-wave laser beams ${ }^{1}$. A region of $K \leq M$ sites is coupled to two quantized light modes whose geometries (that is, axis directions or wavelengths) can be varied. This is shown in Fig. 1, which shows two cavities crossed by a one-dimensional (1D) 
string of atoms in equally separated wells generated by the lattice lasers (not shown). In practice, two different modes of the same cavity would do as well.

As shown in the Methods section, the Heisenberg equations for the annihilation operators of two light modes $a_{l}(l=0,1)$ with eigenfrequencies $\omega_{l}$ and spatial mode functions $\boldsymbol{u}_{l}(\mathbf{r})$ are

$$
\begin{gathered}
\dot{a}_{l}=-i\left(\omega_{l}+\delta_{l} \hat{D}_{l l}\right) a_{l}-i \delta_{m} \hat{D}_{l m} a_{m}-\kappa a_{l}+\eta_{l}(t), \\
\text { with } \quad \hat{D}_{l m} \equiv \sum_{i=1}^{K} u_{l}^{*}\left(\mathbf{r}_{i}\right) u_{m}\left(\mathbf{r}_{i}\right) \hat{n}_{i},
\end{gathered}
$$

where $l \neq m, \delta_{l}=g^{2} / \Delta_{\mathrm{la}}, g$ is the atom-light coupling constant, $\Delta_{\mathrm{la}}=\omega_{l}-\omega_{\mathrm{a}}$ are the large cavity-atom detunings, $\kappa$ is the cavity relaxation rate, $\eta_{l}(t)=\eta_{l} \mathrm{e}^{-i \omega_{l p} t}$ gives the external probe and $\hat{n}_{i}$ are the atom number operators at a site with coordinate $\mathbf{r}_{i}$. We also introduce the operator of the atom number at illuminated sites $\hat{N}_{K}=\sum_{i=1}^{K} \hat{n}_{i}$.

In a classical limit, equation (1) corresponds to Maxwell's equations with the dispersion-induced frequency shifts of cavity modes $\delta_{l} \hat{D}_{l l}$ and the coupling coefficient between them $\delta_{1} \hat{D}_{10}$. For a quantum gas, those quantities are operators, which will lead to striking results: atom number fluctuations will be directly reflected in such measurable frequency-dependent observables. Thus, cavity transmission spectra will reflect atomic statistics.

Equation (1) allows us to express the light operators $a_{l}$ as a function $f\left(\hat{n}_{1}, \ldots, \hat{n}_{M}\right)$ of atomic occupation number operators and to calculate their expectation values for prescribed atomic states $|\Psi\rangle$. We start with the well-known examples of MI and SF states and generalize to any $|\Psi\rangle$ later.

From the viewpoint of light scattering, the MI state behaves almost classically as, for negligible tunnelling, precisely $\left\langle\hat{n}_{i}\right\rangle_{\mathrm{MI}}=q_{i}$ atoms are well localized at the $i$ th site with no number fluctuations. It is represented by a product of Fock states, that is, $|\Psi\rangle_{\mathrm{MI}}=\prod_{i=1}^{M}\left|q_{i}\right\rangle_{i} \equiv\left|q_{1}, \ldots, q_{M}\right\rangle$, with expectation values

$$
\left\langle f\left(\hat{n}_{1}, \ldots, \hat{n}_{M}\right)\right\rangle_{\mathrm{MI}}=f\left(q_{1}, \ldots, q_{M}\right),
$$

as $\hat{n}_{i}\left|q_{1}, \ldots, q_{M}\right\rangle=q_{i}\left|q_{1}, \ldots, q_{M}\right\rangle$. For simplicity, we consider equal average densities $\left\langle\hat{n}_{i}\right\rangle_{\mathrm{MI}}=N / M \equiv n\left(\left\langle\hat{N}_{K}\right\rangle_{\mathrm{MI}}=n K \equiv N_{K}\right)$.

In our second example, the SF state, each atom is delocalized over all sites leading to local number fluctuations at a lattice region with $K<M$ sites. Mathematically it is a superposition of Fock states corresponding to all possible distributions of $N$ atoms at $M$ sites: $|\Psi\rangle_{\mathrm{SF}}=\sum_{q_{1}, \ldots, q_{M}} \sqrt{N ! / M^{N}} / \sqrt{q_{1} ! \cdots q_{M} !}\left|q_{1}, \ldots, q_{M}\right\rangle$. Although its average density $\left\langle\hat{n}_{i}\right\rangle_{\mathrm{SF}}=N / M$ is identical to an MI, it creates different light transmission spectra. Expectation values of light operators can be calculated from

$$
\left\langle f\left(\hat{n}_{1}, \ldots, \hat{n}_{M}\right)\right\rangle_{\mathrm{SF}}=\frac{1}{M^{N}} \sum_{q_{1}, \ldots, q_{M}} \frac{N !}{q_{1} ! \cdots q_{M} !} f\left(q_{1}, \ldots, q_{M}\right),
$$

representing a sum of all possible 'classical' terms. Thus, all these distributions contribute to scattering from an SF, which is obviously different from $\left\langle f\left(\hat{n}_{1}, \ldots, \hat{n}_{M}\right)\right\rangle_{\mathrm{MI}}(2)$ with only a single contributing term.

In the simple case of only one mode $a_{0}\left(a_{1} \equiv 0\right)$, the stationary solution of equation (1) for the photon number reads

$$
a_{0}^{\dagger} a_{0}=f\left(\hat{n}_{1}, \ldots, \hat{n}_{M}\right)=\frac{\left|\eta_{0}\right|^{2}}{\left(\Delta_{\mathrm{p}}-\delta_{0} \hat{D}_{00}\right)^{2}+\kappa^{2}},
$$

where $\Delta_{\mathrm{p}}=\omega_{0 p}-\omega_{0}$ is the probe-cavity detuning. We present calculated transmission spectra in Fig. 2 for the case where
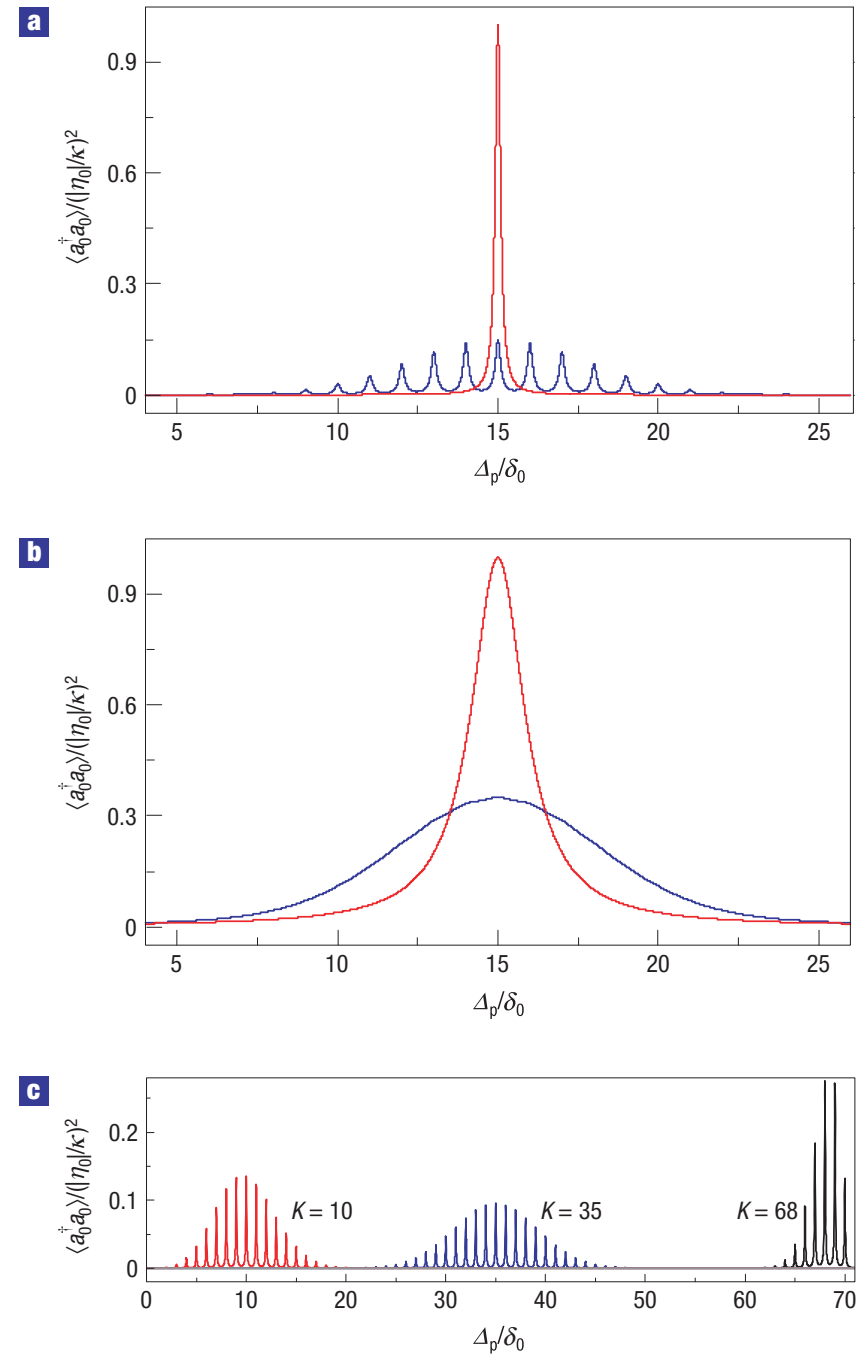

Figure 2 Photon number in a single-cavity mode. a, The single lorentzian for Ml (red line) reflects the non-fluctuating atom number. Many lorentzians for SF (blue line) reflect atom number fluctuations, which are imprinted on the positions of narrow resonances. Here, $\kappa$ is smaller than the satellite separation $\delta_{0}\left(\kappa=0.1 \delta_{0}\right)$, $N=M=30, K=15$. b. The same as in a but $\kappa=\delta_{0}$ gives a smooth broadened contour for SF. c, Spectra for SF with $N=M=70$ and different number of sites illuminated $K=10,35,68$. The transmission spectra have different forms, as different atom distribution functions correspond to different $K . \kappa=0.05 \delta_{0}$.

$\left|u_{0}\left(\mathbf{r}_{i}\right)\right|^{2}=1$ and $\hat{D}_{00}=\sum_{i=1}^{K} \hat{n}_{i}$ reduces to $\hat{N}_{K}$. For a $1 D$ lattice (see Fig. 1), this occurs for a travelling wave at any angle and a standing wave transverse $\left(\theta_{0}=\pi / 2\right)$ or parallel $\left(\theta_{0}=0\right)$ to the lattice with atoms trapped at field maxima.

For an MI, the averaging of equation (4) according to equation (2) gives the photon number $\left\langle a_{0}^{\dagger} a_{0}\right\rangle_{\mathrm{MI}}$, as a function of the detuning, as a single lorentzian described by equation (4) with width $\kappa$ and frequency shift given by $\delta_{0}\left\langle\hat{D}_{00}\right\rangle_{\mathrm{MI}}$ (equal to $\delta_{0} N_{K}$ in Fig. 2). Thus, for an MI, the spectrum reproduces a simple classical result of a lorentzian shifted owing to dispersion.

In contrast, for an SF, the averaging procedure of equation (3) gives a sum of lorentzians with different dispersion shifts corresponding to all atomic distributions $\left|q_{1}, \ldots, q_{K}\right\rangle$. So, if each lorentzian is resolved, we can measure a comb-like structure by scanning the detuning $\Delta_{\mathrm{p}}$. In Fig. 2a,c, different shifts of the 

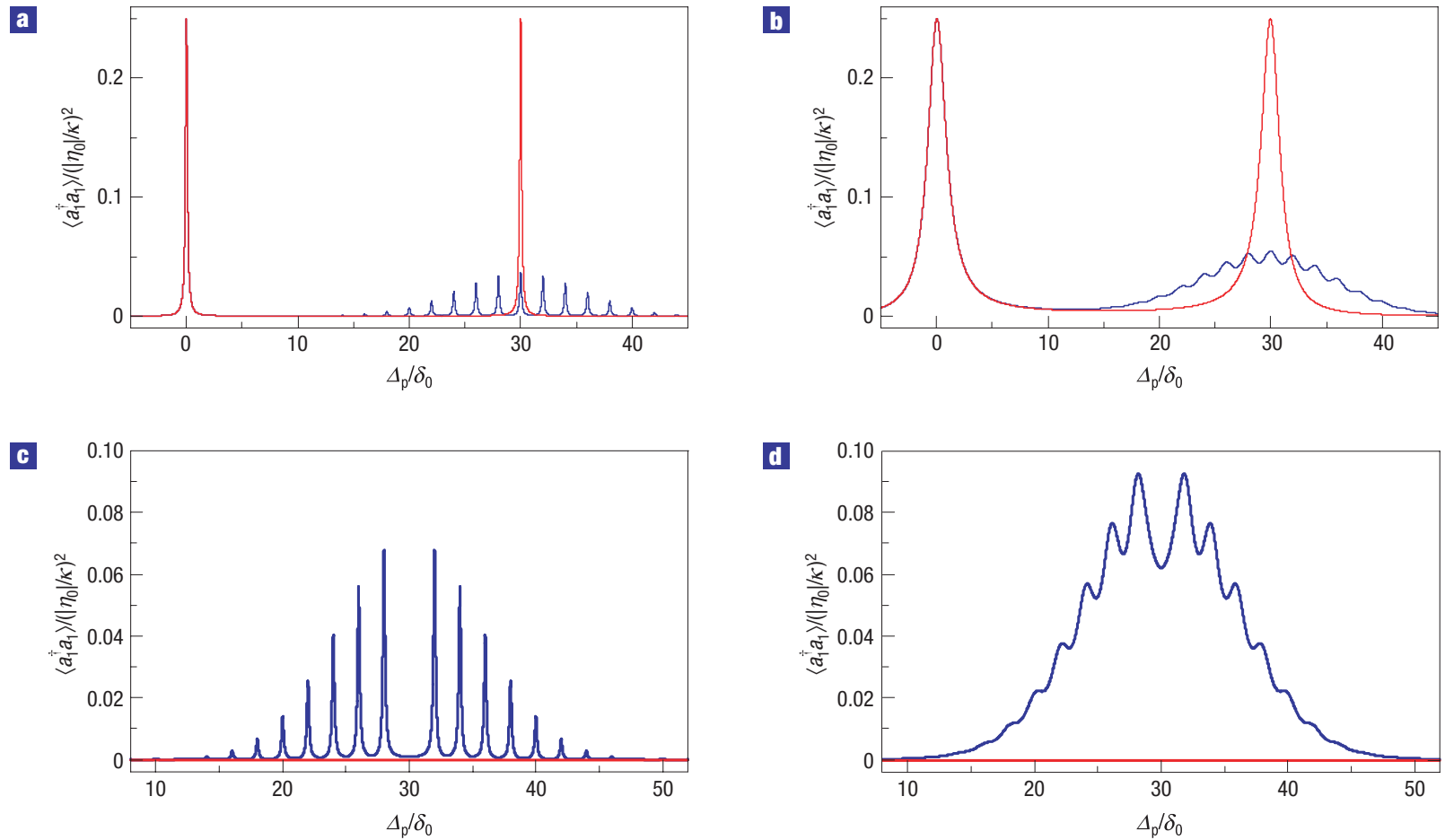

Figure 3 Photon number in one of two strongly coupled modes. a, Diffraction maximum, doublet for Ml (red line) and spectrum with a structured right satellite for SF (blue line). Left satellites at zero detuning coincide for MI and SF. The structure in the satellite reflects atom number fluctuations in SF, whereas the narrow spectrum for MI demonstrates vanishing fluctuations. Here, $\kappa$ is smaller than the satellite separation $2 \delta_{0}\left(\kappa=0.1 \delta_{0}\right), K=15$. $\mathbf{b}$, The same as in a but $\kappa=\delta_{0}$ gives a broadened satellite for SF. c, Diffraction minimum, zero field for Ml and structured spectrum for SF. The non-zero structured spectrum for SF reflects the fluctuating difference between atom numbers at odd and even sites, which exists even for the whole lattice illuminated, $K=M$. Here, $\kappa$ is smaller than the satellite separation $2 \delta_{0}\left(\kappa=0.1 \delta_{0}\right), K=30$. $\mathbf{d}$, The same as in $\mathbf{c}$ but $\kappa=\delta_{0}$ gives a broadened contour for SF. $N=M=30$ in all figures.

lorentzians correspond to different possible atom numbers at $K$ sites (which owing to atom number fluctuations in SF, can take all values $0,1,2, \ldots, N)$. The lorentzians are separated by $\delta_{0}$. Thus, we see that atom number fluctuations lead to the fluctuating mode shift, and hence to multiple resonances in the spectrum. For larger $\kappa$, the spectrum becomes continuous (Fig. 2b), but broader than that for MI.

Scattering of weak fields does not change the atom number distribution. However, as the SF is a superposition of different atom numbers in a region with $K$ sites, a measurement projects the state into a subspace with fixed $N_{K}$ in this region, and a subsequent measurement on a timescale shorter than tunnelling between sites will yield the same result. The full spectrum of Fig. 2 is recovered by repeating the experiment or with sufficient delay to allow for redistribution via tunnelling. Such measurements will allow a time-dependent study of tunnelling and build-up of longrange order. Alternatively, measurements can be continued on the reduced subspace after changing a lattice region or light geometry.

We now consider two modes with $\omega_{0}=\omega_{1}$, the probe injected only into $a_{0}$ (Fig. 1) and the mentioned geometries where $\hat{D}_{00}=\hat{D}_{11}=\hat{N}_{K}$ (see Fig. 3 ). From equation (1), the stationary photon number $a_{1}^{\dagger} a_{1}=f\left(\hat{n}_{1}, \ldots, \hat{n}_{M}\right)$ is

$$
a_{1}^{\dagger} a_{1}=\frac{\delta_{1}^{2} \hat{D}_{10}^{\dagger} \hat{D}_{10}\left|\eta_{0}\right|^{2}}{\left[\hat{\Delta}_{\mathrm{p}}^{2}-\delta_{1}^{2} \hat{D}_{10}^{\dagger} \hat{D}_{10}-\kappa^{2}\right]^{2}+4 \kappa^{2} \hat{\Delta}_{\mathrm{p}}^{\prime 2}},
$$

where $\hat{\Delta}_{\mathrm{p}}^{\prime}=\Delta_{\mathrm{p}}-\delta_{1} \hat{D}_{11}$.

In a classical (and the MI) case, equation (2) gives a twosatellite contour (5) reflecting normal-mode splitting of two oscillators $\left\langle a_{0,1}\right\rangle$ coupled through atoms. This was recently observed $^{21}$ for collective strong coupling, that is, the splitting $\delta_{1}\left\langle\hat{D}_{10}\right\rangle$ exceeding $\kappa$. The splitting depends on the geometry (see equation (1)) representing diffraction of one mode into another. Thus, our results can be treated as scattering from a 'quantum diffraction grating' generalizing Bragg scattering, well known in different disciplines. In diffraction maxima (that is, $\left.u_{1}^{*}\left(\mathbf{r}_{i}\right) u_{0}\left(\mathbf{r}_{i}\right)=1\right)$ we find $\hat{D}_{10}=\hat{N}_{K}$ providing the maximal classical splitting. In diffraction minima, we find $\hat{D}_{10}=\sum_{i=1}^{K}(-1)^{i+1} \hat{n}_{i}$ providing both the classical splitting and photon number are almost zero.

In an SF, equation (3) shows that $\left\langle a_{1}^{\dagger} a_{1}\right\rangle_{\mathrm{SF}}$ is given by a sum of all classical terms with all possible normal-mode splittings. In a diffraction maximum (Fig. 3a,b), the right satellite is split into components corresponding to all possible $N_{K}$ or extremely broadened. In a minimum (Fig. $3 \mathrm{c}, \mathrm{d}$ ), the splittings are determined by all differences between atom numbers at odd and even sites $\sum_{i=1}^{K}(-1)^{i+1} q_{i}$. Note that there is no classical description of the spectra in a minimum, because here the classical field (and $\left.\left\langle a_{1}^{\dagger} a_{1}\right\rangle_{\mathrm{MI}}\right)$ is simply zero for any $\Delta_{\mathrm{p}}$. Thus, for two cavities coupled at the diffraction minimum, the difference between the SF and MI states is even more striking: one has a structured spectrum instead of zero signal. Moreover, the difference between atom numbers at odd and even sites fluctuates even for the whole lattice illuminated, giving non-trivial spectra even for $K=M$.

In each of the examples in Figs 2 and 3, the photon number depends only on one statistical quantity, now called $q$, $f\left(q_{1}, \ldots, q_{M}\right)=f(q)$. For the single mode and two modes in a maximum, $q$ is the atom number at $K$ sites. For two modes in a minimum, $q$ is the atom number at odd (or even) sites. 
Therefore, expectation values for some state $|\Psi\rangle$ can be reduced to $\langle f\rangle_{\Psi}=\sum_{q=0}^{N} f(q) p_{\Psi}(q)$, where $p_{\Psi}(q)$ is the distribution function of $q$ in this state.

In high- $Q$ cavities $\left(\kappa \ll \delta_{0}=g^{2} / \Delta_{0 a}\right), f(q)$ is given by a narrow lorentzian of width $\kappa$ peaked at some frequency proportional to $q$ $(q=0,1, \ldots, N)$. The lorentzian height is independent of $q$. Thus, $\langle f\rangle_{\Psi}$ as a function of $\Delta_{\mathrm{p}}$ represents a comb of lorentzians with the amplitudes simply proportional to $p_{\Psi}(q)$.

This is our central result. It states that the transmission spectrum of a high-Q cavity $\left\langle a^{\dagger} a\left(\Delta_{\mathrm{p}}\right)\right\rangle_{\Psi}$ directly maps the distribution function of ultracold atoms $p_{\Psi}(q)$, for example, the distribution function of atom number at $K$ sites. Various atomic statistical quantities characterizing a particular state can be then calculated: mean value (given by the spectrum centre), variance (determined by the spectral width) and higher moments. Furthermore, transitions between different states will be reflected in spectral changes. Deviations from idealized MI and SF states ${ }^{22}$ are also measurable.

For an SF, using $p_{\mathrm{SF}}(q)$ (see the Methods section), we can write the envelopes of the comb of lorentzians shown in Figs 2a,c and 3a,c. As known, the atom number at $K$ sites fluctuates in an SF with the variance $\left(\Delta N_{K}\right)^{2}=N_{K}(1-K / M)$. For example, Fig. $2 \mathrm{c}$ shows spectra for different lattice regions demonstrating gaussian and poissonian distributions with the spectral width $\sigma_{\omega}=\delta_{0} \sqrt{\left(\Delta N_{K}\right)^{2}}$, directly reflecting the atom distribution functions in an SF. For $K \approx M$, the spectrum narrows and, for the whole lattice illuminated, shrinks to a single lorentzian as in an MI.

The condition $\kappa<\delta_{0}=g^{2} / \Delta_{0 a}$ is already met in present experiments. In recent work $^{8}$, where set-ups of cavity QED and ultracold gases were joined to probe quantum statistics of an atom laser with ${ }^{87} \mathrm{Rb}$ atoms, the parameters were $\left(g, \Delta_{0 a}, \kappa\right)=$ $2 \pi \times(10.4,30,1.4) \mathrm{MHz}$. The set-ups of cavity cooling ${ }^{23,24}$ are also very promising.

For bad cavities $\left(\kappa \gg \delta_{0}=g^{2} / \Delta_{0 a}\right)$, the sums can be replaced by integrals. The broad spectra in Figs $2 \mathrm{~b}$ and $3 \mathrm{~b}, \mathrm{~d}$ are then given by convolutions of $p_{\Psi}(q)$ and lorentzians. For example, the blue line in Fig. 2 b represents a Voigt contour, well known in spectroscopy of hot gases. Here, the 'inhomogeneous broadening' is a striking contribution of quantum statistics.

In summary, we showed that transmission spectra of cavities around a degenerate gas in an optical lattice are distinct for different quantum phases of equal densities. Similar information is also contained in the field amplitudes $\left\langle a_{0,1}\right\rangle$, in contrast to previous suggestions ${ }^{13}$ that $\left\langle a_{0,1}\right\rangle$ probes only the average density. This reflects (1) the orthogonality of Fock states corresponding to different atom distributions and (2) the different frequency shifts of light fields entangled to those states. In general, other optical phenomena and quantities depending nonlinearly on atom number operators should similarly reflect the underlying quantum statistics ${ }^{25-27}$.

\section{METHODS}

\section{DERIVATION OF HEISENBERG EQUATIONS}

A many-body hamiltonian for our system shown in Fig. 1 is given by

$$
\begin{gathered}
H=\sum_{l=0,1} \hbar \omega_{l} a_{l}^{\dagger} a_{l}+\int \mathrm{d}^{3} \mathbf{r} \Psi^{\dagger}(\mathbf{r}) H_{\mathrm{a} 1} \Psi(\mathbf{r}), \quad \text { with } \\
H_{\mathrm{a} 1}=\frac{\mathbf{p}^{2}}{2 m_{\mathrm{a}}}+V_{\mathrm{cl}}(\mathbf{r})+\hbar g^{2} \sum_{l, m=0,1} \frac{u_{l}^{*}(\mathbf{r}) u_{m}(\mathbf{r}) a_{l}^{\dagger} a_{m}}{\Delta_{m a}},
\end{gathered}
$$

where $a_{0,1}$ are the annihilation operators of the modes of frequencies $\omega_{0,1}$, wavevectors $\mathbf{k}_{0,1}$ and mode functions $u_{0,1}(\mathbf{r}) ; \Psi(\mathbf{r})$ is the atom-field operator. In the effective single-atom hamiltonian $H_{\mathrm{a} 1}, \mathbf{p}$ and $\mathbf{r}$ are the momentum and position operators of an atom of mass $m_{\mathrm{a}}$ trapped in the classical potential
$V_{\mathrm{cl}}(\mathbf{r})$ and $g$ is the atom-light coupling constant. We consider off-resonant scattering where the detunings between fields and atomic transition $\Delta_{\mathrm{la}}=\omega_{l}-\omega_{\mathrm{a}}$ are larger than the spontaneous emission rate and Rabi frequencies. Thus, in $H_{\mathrm{a} 1}$, the adiabatic elimination of the upper state, assuming linear dipoles with polarization following adiabatically, was used.

For a 1D lattice with period $d$ and atoms trapped at $x_{j}=j d$

$(j=1,2, \ldots, M)$, the mode functions are $u_{0,1}\left(\mathbf{r}_{j}\right)=\exp \left(i j k_{0,1 x} d+i \phi\right)$ for travelling and $u_{0,1}\left(\mathbf{r}_{j}\right)=\cos \left(j k_{0,1 x} d+\phi\right)$ for standing waves where

$k_{0,1 x}=\left|\mathbf{k}_{0,1}\right| \cos \theta_{0,1}, \theta_{0,1}$ are angles between the mode and lattice axes and $\phi$ is some spatial phase shift (see Fig. 1).

Assuming that the modes $a_{0,1}$ are much weaker than the trapping beam, we expand $\Psi(\mathbf{r})$ using localized Wannier functions ${ }^{7}$ corresponding to the potential $V_{\mathrm{cl}}(\mathbf{r})$ and keep only the lowest vibrational state at each site (we consider a quantum degenerate gas): $\Psi(\mathbf{r})=\sum_{i=1}^{M} b_{i} w\left(\mathbf{r}-\mathbf{r}_{i}\right)$, where $b_{i}$ is the annihilation operator of an atom at site $i$ at a position $\mathbf{r}_{i}$. Substituting this expansion in the hamiltonian $H$, we can get a generalized Bose-Hubbard model $^{7}$ including light scattering. In contrast to 'Bragg spectroscopy', which involves scattering of matter waves ${ }^{4}$, and our previous work ${ }^{28}$, we neglect lattice excitations here and focus on light scattering from atoms in some prescribed quantum states.

Neglecting atomic tunnelling, the hamiltonian reads:

$$
H=\sum_{l=0,1} \hbar \omega_{l} a_{l}^{\dagger} a_{l}+\hbar g^{2} \sum_{l, m=0,1} \frac{a_{l}^{\dagger} a_{m}}{\Delta_{m a}}\left(\sum_{i=1}^{K} J_{i, i}^{l m} \hat{n}_{i}\right),
$$

where $\hat{n}_{i}=b_{i}^{\dagger} b_{i}$. For deep lattices, the coefficients

$J_{i, i}^{l m}=\int \mathrm{d} \mathbf{r} w^{2}\left(\mathbf{r}-\mathbf{r}_{i}\right) u_{l}^{*}(\mathbf{r}) u_{m}(\mathbf{r})$ reduce to $J_{i, i}^{l m}=u_{l}^{*}\left(\mathbf{r}_{i}\right) u_{m}\left(\mathbf{r}_{i}\right)$ neglecting spreading of atoms, which can be characterized even by classical scattering ${ }^{29}$. The Heisenberg equations obtained from this hamiltonian are given by equation (1), where we have added a relaxation term. Strictly speaking, a Langevin noise term should also be added to equation (1). However, for typical conditions, its influence on the expectation values of normal ordered field operators is negligible (see, for example, ref. 30). Here, we are interested only in the number of photons $\left\langle a_{l}^{\dagger} a_{l}\right\rangle$, which is a normal ordered quantity. Thus, we can simply omit the noise term in equation (1).

\section{SIMPLE EXPRESSIONS FOR SPECTRAL LINE SHAPES IN THE SF STATE}

We will now derive expressions for the spectra shown in Figs 2 and 3 demonstrating relations between atomic quantum statistics and the transmission spectra for the SF state. As mentioned in the main text, in all examples shown in Figs 2 and 3, the photon number depends only on a single statistical quantity, which we denote as $q$. Using this fact, the multinomial distribution in equation (3) reduces to a binomial, which can be directly derived from equation (3): $\langle f\rangle_{\mathrm{SF}}=\sum_{q=0}^{N} f(q) p_{\mathrm{SF}}(q)$ with $p_{\mathrm{SF}}(q)=N ! /[q !(N-q) !](Q / M)^{q}(1-Q / M)^{N-q}$ and a single sum instead of $M$ ones. Here, $Q$ is the number of specified sites: $Q$ is equal to $K$ for one mode and two modes in a maximum; $Q$ is the number of odd (or even) sites for two modes in a minimum $(Q=M / 2$ for even $M)$. This approach can be used for other geometries, for example, for two modes in a minimum and $K<M$, where equation (3) can be reduced to a trinomial distribution.

As a next approximation, we consider $N, M \gg 1$, but finite $N / M$, leading to the gaussian distribution $p_{\mathrm{SF}}(q)=1 /\left(\sqrt{2 \pi} \sigma_{q}\right) \exp \left[-(q-\tilde{q})^{2} / 2 \sigma_{q}^{2}\right]$ with central value $\tilde{q}=N Q / M$ and width $\sigma_{q}=\sqrt{N(Q / M)(1-Q / M)}$.

In high- $Q$ cavities $\left(\kappa \ll \delta_{0}=g^{2} / \Delta_{0 a}\right), f(q)$ is a narrow lorentzian of width $\kappa$ peaked at some $q$-dependent frequency, now called $\Delta_{\mathrm{p}}^{q}$. As the lorentzian height is $q$ independent, $\langle f\rangle_{\mathrm{SF}}$ as a function of $\Delta_{\mathrm{p}}$ is a comb of lorentzians with the amplitudes proportional to $p_{\mathrm{SF}}(q)$.

Using the gaussian distribution $p_{\mathrm{SF}}(q)$, we can write the envelope of such a comb. For a single mode (Fig. 2a,c and equation (4)), we find $\Delta_{\mathrm{p}}^{q} \approx \delta_{0} q$ with the envelope

$$
\left\langle a_{0}^{\dagger} a_{0}\left(\Delta_{\mathrm{p}}^{q}\right)\right\rangle_{\mathrm{SF}}=\frac{\alpha \delta_{0}}{\sqrt{2 \pi} \sigma_{\omega}} \mathrm{e}^{-\left(\Delta_{\mathrm{p}}^{q}-\tilde{\Delta}_{\mathrm{p}}\right)^{2} / 2 \sigma_{\omega}^{2}}
$$

where the central frequency $\tilde{\Delta}_{\mathrm{p}}=\delta_{0} N_{K}$, spectral width $\sigma_{\omega}=\delta_{0} \sqrt{N_{K}(1-K / M)}$ and $\alpha=\left|\eta_{0}\right|^{2} / \kappa^{2}$. So, the spectrum envelopes in Fig. 2a,c are well described by gaussians of widths strongly depending on $K$.

For $K \rightarrow 0$ and $K \rightarrow M$, the binomial distribution $p_{\mathrm{SF}}(q)$ is well approximated by a poissonian distribution, which is demonstrated in Fig. $2 \mathrm{c}$ for $K=10$ and $K=68$. For $K=M$, the spectrum shrinks to a single lorentzian, as the total atom number at $M$ sites does not fluctuate. 
In other examples (Fig. 3a,c), the above expression is also valid, although with other parameters. For two modes in a diffraction maximum (Fig. 3a), the central frequency, separation between lorentzians and width are doubled: $\tilde{\Delta}_{\mathrm{p}}=2 \delta_{0} N_{K}, \Delta_{\mathrm{p}}^{q} \approx 2 \delta_{0} q$ and $\sigma_{\omega}=2 \delta_{0} \sqrt{N_{K}(1-K / M)} ; \alpha=\left|\eta_{0}\right|^{2} /\left(2 \kappa^{2}\right)$. The left satellite at $\Delta_{\mathrm{p}}=0$ has a classical amplitude $\left|\eta_{0}\right|^{2} /\left(4 \kappa^{2}\right)$.

The non-classical spectrum for two waves in a diffraction minimum (Fig. 3c) is centred at $\tilde{\Delta}_{\mathrm{p}}=\delta_{0} N$, with components at $\Delta_{\mathrm{p}}^{q} \approx 2 \delta_{0} q$, and is very broad, $\sigma_{\omega}=\delta_{0} \sqrt{N} ; \alpha=\left|\eta_{0}\right|^{2} / \kappa^{2}$.

For bad cavities $\left(\kappa \gg \delta_{0}\right)$, the sums can be replaced by integrals with the same parameters $\tilde{\Delta}_{\mathrm{p}}$ and $\sigma_{\omega}$ as for $\kappa<\delta_{0}$. For a single mode, Fig. $2 \mathrm{~b}$ represents a Voigt contour

$$
\left\langle a_{0}^{\dagger} a_{0}\left(\Delta_{\mathrm{p}}\right)\right\rangle_{\mathrm{SF}}=\frac{\left|\eta_{0}\right|^{2}}{\sqrt{2 \pi} \sigma_{\omega}} \int_{0}^{\infty} \frac{\mathrm{e}^{-\left(\omega-\tilde{\Delta_{\mathrm{p}}}\right)^{2} / 2 \sigma_{\omega}^{2}} \mathrm{~d} \omega}{\left(\Delta_{\mathrm{p}}-\omega\right)^{2}+\kappa^{2}} .
$$

For two modes in a diffraction minimum, the photon number (Fig. 3d) is

$$
\left\langle a_{1}^{\dagger} a_{1}\right\rangle_{\mathrm{SF}}=\frac{\left|\eta_{0}\right|^{2}}{\sqrt{2 \pi} \sigma_{\omega}} \int_{-\infty}^{\infty} \frac{\omega^{2} \mathrm{e}^{-\omega^{2} / 2 \sigma_{\omega}^{2}} \mathrm{~d} \omega}{\left(\Delta_{\mathrm{p}}^{\prime 2}-\omega^{2}-\kappa^{2}\right)^{2}+4 \kappa^{2} \Delta_{\mathrm{p}}^{\prime 2}},
$$

where $\Delta_{\mathrm{p}}^{\prime}=\Delta_{\mathrm{p}}-\tilde{\Delta}_{\mathrm{p}}$, whereas in a maximum (Fig. 3b)

$$
\left\langle a_{1}^{\dagger} a_{1}\right\rangle_{\mathrm{SF}}=\frac{\left|\eta_{0}\right|^{2}}{4 \sqrt{2 \pi} \sigma_{\omega}} \int_{0}^{\infty} \frac{\omega^{2} \mathrm{e}^{-\left(\omega-\tilde{\Delta}_{\mathrm{p}}\right)^{2} / 2 \sigma_{\omega}^{2}} \mathrm{~d} \omega}{\left[\Delta_{\mathrm{p}}\left(\Delta_{\mathrm{p}}-\omega\right)+\kappa^{2}\right]^{2}+\kappa^{2} \omega^{2}} .
$$

Received 10 November 2006; accepted 19 February 2007; published 25 March 2007.

\section{References}

1. Bloch, I. Ultracold quantum gases in optical lattices. Nature Phys. 1, 23-30 (2005).

2. Fölling, S. et al. Spatial quantum noise interferometry in expanding ultracold atom clouds. Nature 434, 481-484 (2005)

3. Altman, E., Demler, E. \& Lukin, M. D. Probing many-body states of ultracold atoms via noise correlations. Phys. Rev. A 70, 013603 (2004)

4. Stöferle, T., Moritz, H., Schori, C., Köhl, M. \& Esslinger, T. Transition from a strongly interacting 1D superfluid to a Mott insulator. Phys. Rev. Lett. 92, 130403 (2004).

5. Gritsev, V., Altman, E., Demler, E. \& Polkovnikov, A. Full quantum distribution of contrast in interference experiments between interacting one-dimensional Bose liquids. Nature Phys. 2, 705-709 (2006).

6. Schellekens, M. et al. Hanbury Brown Twiss effect for ultracold quantum gases. Science $\mathbf{3 1 0}$ 648-651 (2005)

7. Jaksch, D., Bruder, C., Cirac, J. I., Gardiner, C. W. \& Zoller, P. Cold bosonic atoms in optical lattices. Phys. Rev. Lett. 81, 3108-3111 (1998).

8. Bourdel, T. et al. Cavity QED detection of interfering matter waves. Phys. Rev. A 73, 043602 (2006).

9. You, L., Lewenstein, M. \& Cooper, J. Line shapes for light scattered from Bose-Einstein condensates. Phys. Rev. A 50, R3565-R3568 (1994).
10. Javanainen, J. Optical signatures of a tightly confined Bose condensate. Phys. Rev. Lett. 72, 2375-2378 (1994).

11. You, L., Lewenstein, M. \& Cooper, J. Quantum field theory of atoms interacting with photons. II. Scattering of short laser pulses from trapped bosonic atoms. Phys. Rev. A 51, $4712-4727$ (1995).

12. Javanainen, J. \& Ruostekoski, J. Off-resonance light scattering from low-temperature Bose and Fermi gases. Phys. Rev. A 52, 3033-3046 (1995).

13. Parkins, A. S. \& Walls, D. F. The physics of trapped dilute-gas Bose-Einstein condensates. Phys. Rep. 303, 1-80 (1998).

14. Morice, O., Castin, Y. \& Dalibard, J. Refractive index of a dilute Bose gas. Phys. Rev. A 51, 3896-3901 (1995).

15. Brune, M. et al. Quantum Rabi oscillation: a direct test of field quantization in a cavity. Phys. Rev. Lett. 76, 1800-1803 (1996)

16. Gambetta, J. et al. Qubit-photon interactions in a cavity: Measurement-induced dephasing and number splitting. Phys. Rev. A 74, 042318 (2006).

17. Campbell, G. K. et al. Imaging the Mott insulator shells by using atomic clock shifts. Science 313, 649-652 (2006).

18. Gerbier, F., Fölling, S., Widera, A., Mandel, O. \& Bloch, I. Probing number squeezing of ultracold atoms across the superfluid-Mott insulator transition. Phys. Rev. Lett. 96, 090401 (2006).

19. Volz, T. et al. Preparation of a quantum state with one molecule at each site of an optical lattice. Nature Phys. 2, 692-695 (2006).

20. Winkler, K. et al. Repulsively bound atom pairs in an optical lattice. Nature 441, 853-856 (2006).

21. Klinner, J., Lindholdt, M., Nagorny, B. \& Hemmerich, A. Normal mode splitting and mechanical effects of an optical lattice in a ring cavity. Phys. Rev. Lett. 96, 023002 (2006).

22. Lewenstein, M. et al. Ultracold atomic gases in optical lattices: Mimicking condensed matter physics and beyond. Adv. Phys. (in the press); preprint at $<$ http://www.arxiv.org/cond-mat/0606771> (2006).

23. Maunz, P. et al. Cavity cooling of a single atom. Nature 428, 50-52 (2004).

24. Hood, C. J., Lynn, T. W., Doherty, A. C., Parkins, A. S. \& Kimble, H. J. The atom-cavity microscope: Single atoms bound in orbit by single photons. Science 287, 1447-1453 (2000).

25. Mekhov, I. B., Maschler, C. \& Ritsch, H. Cavity enhanced light scattering in optical lattices to probe atomic quantum statistics. Phys. Rev. Lett. 98, 100402 (2007).

26. Mekhov, I. B., Maschler, C. \& Ritsch, H. Books of Abstracts for the XX International Conference on Atomic Physics, ICAP 309 (Institute for Quantum Optics and Quantum Information, Innsbruck, 2006).

27. Chen, W., Meiser, D. \& Meystre, P. Cavity QED determination of atomic number statistics in optical lattices. Phys. Rev. A 75, 023812 (2007).

28. Maschler, C. \& Ritsch, H. Cold atom dynamics in a quantum optical lattice potential. Phys. Rev. Lett. 95, 260401 (2005).

29. Slama, S., von Cube, C., Kohler, M., Zimmermann, C. \& Courteille, P. V. Multiple reflections and diffuse scattering in Bragg scattering at optical lattices. Phys. Rev. A 73, 023424 (2006).

30. Davidovich, L. Sub-Poissonian processes in quantum optics. Rev. Mod. Phys. 68, 127 (1996).

\section{Acknowledgements}

The work was supported by FWF (P17709 and S1512). While preparing this manuscript, we became aware of a closely related study in the group of P. Meystre. We are grateful to him for sending us the preprint $^{27}$ and stimulating discussions.

Correspondence and requests for materials should be addressed to I.B.M. or H.R.

\section{Competing financial interests}

The authors declare no competing financial interests.

Reprints and permission information is available online at http://npg.nature.com/reprintsandpermissions/ 